\title{
Computing the Kirkwood g-factor by combining constant Maxwell electric field and electric displacement simulations: Application to the dielectric constant of liquid water
}

\author{
Chao Zhang ${ }^{\dagger}$, Jürg Hutter ${ }^{\ddagger}$ and Michiel Sprik ${ }^{\dagger}$ \\ ${ }^{\dagger}$ Department of Chemistry, University of Cambridge, Lensfeld Rd, Cambridge CB2 IEW \\ ${ }^{\ddagger}$ Institut für Chemie, Universität Zürich, Winterthurerstrasse 190, CH-8057 Zürich, Switzerland \\ cz302@cam.ac.uk
}

\section{Supporting Information}

\section{A Polarizable Polar Models}

To compute the static dielectric constant $\epsilon$ of polarizable water models, such as SWM4-DP and PBE, the fluctuation equations Eqs. M3 and M4 for rigid (non-polarizable) molecules must be adjusted for an optical dielectric constant $\epsilon_{\infty} \neq 1$ (Equations in the Main Text will be referred to by capital $M$ followed by the number).

\section{A.1 $\quad \mathbf{E}=0$ boundary condition}

The generalization of Eq. M3 appropriate for standard Ewald summation boundary conditions is given by Eq. M9. This is expression used by several authors and is now accepted as the correct one [1-3]. Converted to fluctuations of the bulk polarization density $\mathbf{P}=\mathbf{M} / \Omega(\Omega$ is the volume of the cubic MD cell) this expression becomes

$$
\frac{4 \pi \beta \Omega}{3}\left(\left\langle\mathbf{P}^{2}\right\rangle_{\mathbf{E}=0}-\langle\mathbf{P}\rangle_{\mathbf{E}=0}^{2}\right)=\epsilon-\epsilon_{\infty}
$$

Eq. 1 was derived by Neumann and Steinhauser in the framework of the reaction field treatment of electrostatic interactions in periodic systems [3]. In our technical publication [4], we presented an alternative derivation of Eq. M3 directly obtaining this expression from the constant electric field Hamiltonian of Stengel, Spaldin and Vanderbilt (SSV) [5]. This derivation can be generalized for a liquid consisting of polar-polarizable molecules reproducing Eq. 1. The key step in this argument is the application of a version of the Hellmann-Feynman theorem.

As in Ref. 4 we consider a periodic system subject to a constant Maxwell electric field $E_{x}$ along the $x$ axis. The SSV Hamiltonian for this system can be written as:

$$
\mathcal{H}_{E}\left(\nu, \varphi, E_{x}\right)=H_{\mathrm{PBC}}(\nu, \varphi)-\Omega E_{x} P_{x}(\nu, \varphi)
$$


where $v=\left(\mathrm{r}^{N}, \mathrm{p}^{N}\right)$ are the classical degrees of freedom of the $N$ atom system. The electronic degrees of freedom $\varphi_{\alpha}, \alpha=1 \ldots M$ determining the induced dipoles are collectively represented by $\varphi$. The observable $P_{x}(\nu, \varphi)$ in Eq. 2 is the $x$ component of the total polarization (permanent plus induced). To simplify notation we have written $P_{x}(\nu, \varphi)$ rather than $P_{x}\left(\mathbf{r}^{N}, \varphi\right)$ with the understanding that there is no dependence on momentum.

The effective Hamiltonian $H_{E}\left(\nu, E_{x}\right)$ driving the classical motion of the atoms is obtained from the Hamiltonian Eq. 2 as the variational minimum in the electronic degrees of freedom (pseudo or real).

$$
\begin{aligned}
H_{E}\left(\nu, E_{x}\right) & =\min _{\varphi} \mathcal{H}_{E}\left(\nu, \varphi, E_{x}\right) \\
& =H_{\mathrm{PBC}}\left(\nu, \varphi_{0}\left(\nu, E_{x}\right)\right)-\Omega E_{x} P_{x}\left(\nu, \varphi_{0}\left(\nu, E_{x}\right)\right)
\end{aligned}
$$

$\varphi_{0}\left(\nu, E_{x}\right)$ is the value of $\varphi$ for which the energy is minimal at given atomic configuration $\nu$ and electric field $E_{x}$. The potential energy function in Eq. 3 can be compared to the BornOppenheimer surface of electronic structure calculation. The polarization in the minimal energy state will be indicated by simply omitting the $\varphi$ argument:

$$
P_{x}\left(\nu, E_{x}\right)=P_{x}\left(\nu, \varphi_{0}\left(\nu, E_{x}\right)\right)
$$

In contrast to the non-polarizable case, polarization in polarizable systems is an implicit function of the field because the electronic state $\varphi_{0}\left(\nu, E_{x}\right)$ varies with the field $E_{x}$. Adopting the compact notation of Eq. 4 we can therefore define a field dependent Ewald Hamiltonian

$$
H_{\mathrm{PBC}}\left(\nu, E_{x}\right)=H_{\mathrm{PBC}}\left(\nu, \varphi_{0}\left(\nu, E_{x}\right)\right)
$$

The thermodynamics generated by the Hamiltonian Eq. 3 is obtained from a field dependent partition function

$$
Z_{E}\left(E_{x}\right)=\int d \nu \exp \left[-\beta H_{E}\left(\nu, E_{x}\right)\right]
$$

with the corresponding free energy function

$$
F\left(E_{x}\right)=-k_{\mathrm{B}} T \ln Z_{E}\left(E_{x}\right)
$$

where $k_{\mathrm{B}}$ is Boltzmann's constant and $T$ the temperature. This is similar to the formalism of a system of rigid dipoles [4] except that as a result of the induced dipole moments also electrostatic interactions have become field dependent $[2,3]$.

The equilibrium polarization $P\left(E_{x}\right)$ is formally defined as the expectation value of the observable Eq. 4 in the canonical ensemble $\exp \left[-\beta H_{E}\right]$. Indicating this average by angular brackets we can write

$$
P\left(E_{x}\right)=\left\langle P_{x}\left(\nu, E_{x}\right)\right\rangle
$$

For rigid (non-polarizable) molecules $P\left(E_{x}\right)$ is related to the free energy by a simple thermodynamic derivative [4]

$$
P\left(E_{x}\right)=-\frac{1}{\Omega} \frac{d F\left(E_{x}\right)}{d E_{x}}
$$

The field is varied at constant temperature, which has been suppressed Eq. 9 in order to keep notation simple. Field derivatives of free energy of polarizable molecules are more involved because now also the electrostatic interactions Eq. 5 in principle contribute. However, Eq. 9 
still holds [6]. The argument is an example of the Hellmann-Feynman principle and we will go through the proof in some detail for later reference. Expanding the free energy derivative

$$
\begin{aligned}
\frac{d F\left(E_{x}\right)}{d E_{x}}= & \int d \nu\left(\frac{\partial}{\partial E_{x}} H_{\mathrm{PBC}}\left(\nu, E_{x}\right)-\Omega E_{x} \frac{\partial}{\partial E_{x}} P_{x}\left(\nu, E_{x}\right)\right) \frac{\exp \left[-\beta H_{E}\left(\nu, E_{x}\right)\right]}{Z_{E}\left(E_{x}\right)} \\
& -\frac{\Omega}{Z_{E}\left(E_{x}\right)} \int d \nu P_{x}\left(\nu, E_{x}\right) \exp \left[-\beta H_{E}\left(\nu, E_{x}\right)\right] \\
= & -\frac{\Omega}{Z_{E}\left(E_{x}\right)} \int d \nu\left(P_{x}\left(\nu, E_{x}\right)-\Pi_{x}\left(\nu, E_{x}\right)\right) \exp \left[-\beta H_{E}\left(\nu, E_{x}\right)\right]
\end{aligned}
$$

where we have introduced the quantity

$$
\Pi_{x}\left(\nu, E_{x}\right)=\frac{1}{\Omega}\left(\frac{\partial}{\partial E_{x}} H_{\mathrm{PBC}}\left(\nu, E_{x}\right)-\Omega E_{x} \frac{\partial}{\partial E_{x}} P_{x}\left(\nu, E_{x}\right)\right)
$$

$\Pi_{x}\left(\nu, E_{x}\right)$ has the physical dimension of polarization density. The desired result Eq. 9 would follow if $\Pi_{x}\left(\nu, E_{x}\right)$ vanishes. Indeed it does. To see this we apply the chain rule to Eq. 5.

$$
\begin{aligned}
\frac{\partial}{\partial E_{x}} H_{\mathrm{PBC}}\left(\nu, E_{x}\right) & =\frac{\partial}{\partial E_{x}} H_{\mathrm{PBC}}\left(\nu, \varphi_{0}\left(\nu, E_{x}\right)\right) \\
& =\left.\sum_{\alpha} \frac{\partial \varphi_{\alpha 0}\left(\nu, E_{x}\right)}{\partial E_{x}} \frac{\partial}{\partial \varphi_{\alpha}} H_{\mathrm{PBC}}(\nu, \varphi)\right|_{\varphi=\varphi_{0}\left(\nu, E_{x}\right)}
\end{aligned}
$$

and similarly for the derivative of polarization $P_{x}\left(\nu, E_{x}\right)$ of Eq. 4. Adding we find

$$
\Omega \Pi_{x}\left(\nu, E_{x}\right)=\left.\sum_{\alpha} \frac{\partial \varphi_{\alpha 0}\left(\nu, E_{x}\right)}{\partial E_{x}}\left(\frac{\partial}{\partial \varphi_{\alpha}} \mathcal{H}_{E}\left(\nu, \phi, E_{x}\right)\right)\right|_{\varphi=\varphi_{0}\left(\nu, E_{x}\right)}
$$

The electronic state $\varphi_{0}\left(\nu, E_{x}\right)$ is a stationary point (Eq. 3) and therefore

$$
\left.\frac{\partial}{\partial \varphi_{\alpha}} \mathcal{H}_{E}\left(\nu, \phi, E_{x}\right)\right|_{\varphi=\varphi_{0}\left(\nu, E_{x}\right)}=0
$$

which holds for all $\varphi_{\alpha}$. Indeed, $\Pi_{x}\left(\nu, E_{x}\right)=0$ and makes no contribution to derivative Eq. 10 .

As for polar non-polarizable systems the dielectric susceptibility and susceptibility are related as

$$
\epsilon-1=4 \pi \chi
$$

with the susceptibility defined as

$$
\chi=\frac{d P\left(E_{x}\right)}{d E_{x}}=\frac{d\left\langle P_{x}\left(\nu, E_{x}\right)\right\rangle}{d E_{x}}
$$

Similar to Eq.10 we carry out the field derivative separating the terms containing expectation values of polarization (or its square) from terms generated by the field dependence of the Hamil- 
tonian. The number of terms is larger, the procedure is the same

$$
\begin{aligned}
\frac{\partial P\left(E_{x}\right)}{\partial E_{x}} & =\frac{\partial}{\partial E_{x}}\left(\frac{\int d \nu P_{x}\left(\nu, E_{x}\right) \exp \left[-\beta H_{E}\left(\nu, E_{x}\right)\right]}{Z_{E}\left(E_{x}\right)}\right) \\
& =\left\langle\frac{\partial P_{x}\left(\nu, E_{x}\right)}{\partial E_{x}}\right\rangle \\
& +\frac{\beta \Omega}{Z_{E}\left(E_{x}\right)} \int d \nu P_{x}\left(\nu, E_{x}\right)\left(P_{x}\left(\nu, E_{x}\right)-\Pi_{x}\left(\nu, E_{x}\right)\right) \exp \left[-\beta\left(H_{E}\left(\nu, E_{x}\right)\right]\right. \\
& -\frac{\beta \Omega\left\langle P_{x}\left(\nu, E_{x}\right)\right\rangle}{Z_{E}\left(E_{x}\right)} \int d \nu\left(P_{x}\left(\nu, E_{x}\right)-\Pi_{x}\left(\nu, E_{x}\right)\right) \exp \left[-\beta\left(H_{E}\left(\nu, E_{x}\right)\right]\right. \\
& =\left\langle\frac{\partial P_{x}\left(\nu, E_{x}\right)}{\partial E_{x}}\right\rangle+\beta \Omega\left(\left\langle P_{x}^{2}\left(\nu, E_{x}\right)\right\rangle-\left\langle P_{x}\left(\nu, E_{x}\right)\right\rangle^{2}\right)
\end{aligned}
$$

where in the last line we have set the "Hellmann-Feynman" polarization $\Pi_{x}\left(\nu, E_{x}\right)$ to zero.

In the second term of Eq. 17 we recognize the expected second moment of the polarization fluctuations. The additional is term due the induced polarization at fixed atomic configuration $\nu$. This contribution will be identified with the high (infinite) frequency dielectric constant $\epsilon_{\infty}$

$$
4 \pi\left\langle\frac{\partial P_{x}\left(\nu, E_{x}\right)}{\partial E_{x}}\right\rangle=\epsilon_{\infty}-1
$$

Substituting Eq. 18 with Eq. 15 in Eq. 17 and we find with

$$
\epsilon=\epsilon_{\infty}+4 \pi \Omega \beta\left(\left\langle P_{x}^{2}\left(\nu, E_{x}\right)\right\rangle-\left\langle P_{x}\left(\nu, E_{x}\right)\right\rangle^{2}\right)
$$

Since the system is isotropic, the fluctuations can be averaged over the three Cartesian directions yielding Eq. 1

\section{A.2 $\quad \mathrm{D}=0$ boundary condition}

The constant D SSV Hamiltonian [5] is in our notation written as

$$
\mathcal{H}_{D}\left(\nu, \varphi, D_{x}\right)=H_{\mathrm{PBC}}(\nu, \varphi)+\frac{\Omega}{8 \pi}\left(D_{x}-4 \pi P_{x}(\nu, \varphi)\right)^{2}
$$

where again we have assumed that the field is directed along the $x$-axis. Minimizing in the electronic degrees of freedom $\varphi$ yields an effective constant $D_{x}$ "Born-Oppenheimer" Hamiltonian, the constant $D$ counterpart of Eq. 3

$$
\begin{aligned}
H_{D}\left(\nu, D_{x}\right) & =\min _{\varphi} \mathcal{H}_{D}\left(\nu, \varphi, D_{x}\right) \\
& \left.=H_{\mathrm{PBC}}\left(\nu, D_{x}\right)\right)+\frac{\Omega}{8 \pi}\left(D_{x}-4 \pi P_{x}\left(\nu, D_{x}\right)\right)^{2}
\end{aligned}
$$

with (compare Eqs. 5 and 4)

$$
\begin{aligned}
P_{x}\left(\nu, D_{x}\right) & =P_{x}\left(\nu, \varphi_{0}\left(\nu, D_{x}\right)\right) \\
H_{\mathrm{PBC}}\left(\nu, D_{x}\right) & =H_{\mathrm{PBC}}\left(\nu, \varphi_{0}\left(\nu, D_{x}\right)\right)
\end{aligned}
$$


$\varphi_{0}\left(\nu, D_{x}\right)$ is the value of $\varphi$ for which the energy is minimal at given atomic configuration $\nu$ and displacement field $D_{x}$. The corresponding partition function is:

$$
Z_{D}\left(D_{x}\right)=\int d \nu \exp \left[-\beta H_{D}\left(\nu, D_{x}\right)\right]
$$

The constrained $E$ electric equation of state Eq. 4 is replaced by a constant $D$ equation of state

$$
P\left(D_{x}\right)=\left\langle P_{x}\left(\nu, D_{x}\right)\right\rangle
$$

where the angular brackets now denote an average over the canonical ensemble generated by the Hamiltonian Eq. 21. The $D_{x}$ derivative is the polarizability

$$
\alpha=\frac{d P\left(D_{x}\right)}{d D_{x}}=\frac{\partial\left\langle P_{x}\left(\nu, D_{x}\right)\right\rangle}{\partial D_{x}}
$$

which is related to the static dielectric constant as (compare Eq. 15)

$$
\frac{\epsilon-1}{\epsilon}=4 \pi \alpha
$$

Similarly, $\epsilon_{\infty}$ is obtained from the "vertical" (fixed configuration) polarizability as

$$
4 \pi\left\langle\frac{\partial P_{x}\left(\nu, D_{x}\right)}{\partial D_{x}}\right\rangle=\frac{\epsilon_{\infty}-1}{\epsilon_{\infty}}
$$

Following the procedure outlined section A.1 we fine an auxiliary Hellmann-Feynman polarization

$$
\Pi_{x}\left(\nu, D_{x}\right)=\frac{1}{\Omega} \frac{\partial}{\partial D_{x}} H_{\mathrm{PBC}}\left(\nu, D_{x}\right)-\left(D_{x}-4 \pi P_{x}\left(\nu, D_{x}\right)\right) \frac{\partial}{\partial D_{x}} P_{x}\left(\nu, D_{x}\right)
$$

$\Pi_{x}\left(\nu, D_{x}\right)$ is the constant $D$ variant of Eq. 11. It enters the expression for the field derivative of the constant $D$ Hamiltonian Eq. 21 as an additional induced polarization term,

$$
\frac{\partial}{\partial D_{x}} H_{D}\left(\nu, D_{x}\right)=\frac{\Omega}{4 \pi}\left(D_{x}-4 \pi P_{x}\left(\nu, D_{x}\right)\right)+\Omega \Pi_{x}\left(\nu, D_{x}\right)
$$

which, however, vanishes at the variational minimum (compare Eq. 14).

$$
\Omega \Pi_{x}\left(\nu, D_{x}\right)=\left.\sum_{\alpha} \frac{\partial \varphi_{\alpha 0}\left(\nu, D_{x}\right)}{\partial D_{x}}\left(\frac{\partial}{\partial \varphi_{\alpha}} \mathcal{H}_{D}\left(\nu, \phi, D_{x}\right)\right)\right|_{\varphi=\varphi_{0}\left(\nu, D_{x}\right)}=0
$$

In the end, the field derivative of the constant $D$ is what what one would have expected for a non-polarizable model,

$$
\frac{\partial}{\partial D_{x}} H_{D}\left(\nu, D_{x}\right)=\frac{\Omega}{4 \pi}\left(D_{x}-4 \pi P_{x}\left(\nu, D_{x}\right)\right)
$$

The derivation for the fluctuation expression of polarizability for the polarizable model is effectively the same as for the non-polarizable model [4]. 
This derivation was not explicitly given in Ref. [4] and for completeness follows below. Expanding the thermodynamic derivative we find

$$
\begin{aligned}
\frac{\partial P\left(D_{x}\right)}{\partial D_{x}} & =\frac{\partial}{\partial D_{x}}\left(\frac{\int d \nu P_{x}\left(\nu, D_{x}\right) \exp \left[-\beta\left(H_{D}\left(\nu, D_{x}\right)\right]\right.}{Z_{D}\left(D_{x}\right)}\right) \\
& =\left\langle\frac{\partial P_{x}\left(\nu, D_{x}\right)}{\partial D_{x}}\right\rangle \\
& -\frac{\beta}{Z_{D}\left(D_{x}\right)} \int d \nu P_{x}\left(\nu, D_{x}\right)\left(\frac{\partial}{\partial D_{x}} H_{D}\left(\nu, D_{x}\right)\right) \exp \left[-\beta\left(H_{D}\left(\nu, D_{x}\right)\right]\right. \\
& +\frac{\beta\left\langle P_{x}\left(\nu, D_{x}\right)\right\rangle}{Z_{D}\left(D_{x}\right)} \int d \nu\left(\frac{\partial}{\partial D_{x}} H_{D}\left(\nu, D_{x}\right)\right) \exp \left[-\beta H_{D}\left(\nu, D_{x}\right)\right]
\end{aligned}
$$

Inserting Eq. 31 gives

$$
\begin{aligned}
\frac{\partial P\left(D_{x}\right)}{\partial D_{x}} & =\left\langle\frac{\partial P_{x}\left(\nu, D_{x}\right)}{\partial D_{x}}\right\rangle \\
& -\frac{\beta \Omega}{4 \pi}\left\langle P_{x}\left(\nu, D_{x}\right)\left(D_{x}-4 \pi P_{x}\left(\nu, D_{x}\right)\right)\right\rangle \\
& +\frac{\beta \Omega}{4 \pi}\left\langle P_{x}\left(\nu, D_{x}\right)\right\rangle\left\langle\left(D_{x}-P_{x}\left(\nu, D_{x}\right)\right)\right\rangle
\end{aligned}
$$

The terms proportional to $D_{x}$ cancel and we obtain

$$
\frac{\partial P\left(D_{x}\right)}{\partial D_{x}}=\left\langle\frac{\partial P_{x}\left(\nu, D_{x}\right)}{\partial D_{x}}\right\rangle+\Omega \beta\left(\left\langle P_{x}^{2}\left(\nu, D_{x}\right)\right\rangle-\left\langle P_{x}\left(\nu, D_{x}\right)\right\rangle^{2}\right)
$$

which substituting Eqs 26 and 27 gives

$$
\frac{\epsilon-\epsilon_{\infty}}{\epsilon \epsilon_{\infty}}=4 \pi \Omega \beta\left(\left\langle P_{x}^{2}\left(\nu, D_{x}\right)\right\rangle-\left\langle P_{x}\left(\nu, D_{x}\right)\right\rangle^{2}\right)
$$

Then because the polarization fluctuations are isotropic we arrive at the fluctuation Eq. M10 for polarizable polar fluids at $\mathbf{D}=0$ :

$$
\frac{4 \pi \beta \Omega}{3}\left(\left\langle\mathbf{P}^{2}\right\rangle_{\mathbf{D}=0}-\langle\mathbf{P}\rangle_{\mathbf{D}=0}^{2}\right)=\frac{\epsilon-\epsilon_{\infty}}{\epsilon \epsilon_{\infty}}
$$

The advantage of the Hellman-Feynman route to the fluctuation equations for polarizable polar models is that this derivation makes no assumptions about a particular model for induced polarization. The induced polarization was described by unspecified electronic variables $\varphi_{\alpha}$. The derivation therefore applies to DFT water as well where the $\varphi_{\alpha}$ are the Kohn-Sham orbitals.

\section{A.3 Kirkwood-Onsager formula and superposition formula}

Multiplying Eq. 1 by a factor 2 and adding it to Eq. 36 gives

$$
\begin{aligned}
\frac{4 \pi \beta \Omega}{3}\left(2\left(\left\langle\mathbf{P}^{2}\right\rangle_{\mathbf{E}=0}-\langle\mathbf{P}\rangle_{\mathbf{E}=0}^{2}\right)+\left\langle\mathbf{P}^{2}\right\rangle_{\mathbf{D}=0}-\langle\mathbf{P}\rangle_{\mathbf{D}=0}^{2}\right) & =\frac{(\epsilon-1)(2 \epsilon+1)}{\epsilon} \\
& -\frac{\left(\epsilon_{\infty}-1\right)\left(2 \epsilon_{\infty}+1\right)}{\epsilon_{\infty}}
\end{aligned}
$$


The combination of static and high frequency dielectric constants on right hand side of the above equation is the correct form of the Kirkwood-Onsager formula for polarizable polar liquids as pointed out by Wertheim [7]. The conclusion is that the procedure to extract the short-ranged Kirkwood g-factor $G_{\mathrm{Kc}}(r)$ for polarizable polar liquids remains unchanged, i.e.:

$$
G_{\mathrm{Kc}}(r)=\frac{2 G_{\mathrm{K}}(r)_{\mathbf{E}=0}+G_{\mathrm{K}}(r)_{\mathbf{D}=0}}{3}
$$

The calculated composite Kirkwood g-factor $G_{\mathrm{Kc}}(r)$ and corresponding $r$-dependent $\epsilon$ for SWM4DP water models are shown in Fig. S1. These results are to be compared to the Kirkwood g-factor $G_{\mathrm{Kc}}(r)$ and the $r$-dependent $\epsilon$ for SPC, TIP3P, TIP4P shown in Fig. S2 to Fig. S4.

\section{B Constant D and Constant E Simulations in CP2K}

\section{B.1 Born Charge}

Born charges are often used as benchmarks in electronic structure calculation [8]. A Born charge is defined as:

$$
Z_{i, z z}^{*(S)}=\frac{\partial f_{i, z}}{\partial S_{z}}
$$

where $f_{i, z}$ is the force of atom $i$ in $z$ direction and $S_{z}$ is the applied periodic field $\left(E_{z}\right.$ or $\left.D_{z}\right)$ in the same direction. The relation between Born charge at constant $\mathrm{E}$ and constant $\mathrm{D}$ for an isotropic system is [8]:

$$
Z_{i, z z}^{*(E)}=\epsilon_{z z}^{\infty} Z_{i, z z}^{*(D)}
$$

Since the optical dielectric constant of water in vacuum is very close to 1 , one would expect that the Born charges of water in vacuum are very similar at constant $D_{z}$ and constant $E_{z}$. We have tested this hypothesis by calculating the Born charge of a water molecule in vacuum using $\mathrm{CP} 2 \mathrm{~K}$. The box size is $12 \AA \times 12 \AA \times 12 \AA$ and the structure of water molecule is taken from the geometry optimization. The results are reported in Tables $\mathrm{S} 1$ and $\mathrm{S} 2$

Table S1: Born charge of oxygen and hydrogen atoms of a $\mathrm{H}_{2} \mathrm{O}$ molecule in vacuum computed using the PBE functional at constant $E_{z}$ field.

\begin{tabular}{c|ccc}
\hline & $E_{z}($ TZV2P, GTH, 320 Ry) & $E_{z}($ TZV2P, GTH, 520 Ry) & $E_{z}\left(\right.$ PW, MT, 70Ry) ${ }^{a}$ \\
\hline $\mathrm{Z}_{\mathrm{O}}^{*}$ & -0.32 & -0.32 & -0.33 \\
$\mathrm{Z}_{\mathrm{H}}^{*}$ & 0.16 & 0.16 & 0.16 \\
\hline
\end{tabular}

a: CPMD v 3.15 .3

Table S2: Born charge of oxygen and hydrogen atoms of a $\mathrm{H}_{2} \mathrm{O}$ molecule at constant $D_{z}$ and constant $E_{z}$ compared.

\begin{tabular}{c|cc}
\hline & $D_{z}$ (TZV2P, GTH, 320 Ry) & $E_{z}$ (TZV2P, GTH, 320 Ry) \\
\hline $\mathrm{Z}_{\mathrm{O}}^{*}$ & -0.32 & -0.32 \\
$\mathrm{Z}_{\mathrm{H}}^{*}$ & 0.16 & 0.16 \\
\hline
\end{tabular}




\section{B.2 Optical Dielectric Constant $\epsilon_{\infty}$}

The optical dielectric constant $\epsilon_{\infty}$ at constant $E_{x}$ was defined in Eq. 18. $\epsilon_{\infty}$ can be calculated directly from the finite field dependence of the polarization according to

$$
\epsilon_{\infty}=1+4 \pi \frac{\partial P_{x}\left(E_{x}\right)}{\partial E_{x}}
$$

where $P_{x}$ is the total polarization in the $x$ direction at fixed geometry and $E_{x}$ is the applied macroscopic field in the same direction. The estimate for liquid water is obtained by averaging the result over a set of configurations sampled from an equilibrium are averaged. Similarly the optical dielectric constant $\epsilon_{\infty}$ at constant $\mathrm{D}$ can be calculated as:

$$
\epsilon_{\infty}=1 /\left(1-4 \pi \frac{\partial P_{x}\left(D_{x}\right)}{\partial D_{x}}\right)
$$

$D_{x}$ is the applied electric displacement in the $x$ direction. The system we used to calculate $\epsilon_{\infty}$ consists of 64 water molecules. The box size is $12.432 \AA \times 12.432 \AA \times 12.432 \AA$ and the density is $0.997 \mathrm{~g} \mathrm{~cm}^{-3}$. The results are listed in Tables S3 and S4. In addition, from Eq. 41 and Eq. 42, we see that the ratio must be again $\epsilon_{\infty}$

$$
\frac{\partial P_{x}\left(E_{x}\right)}{\partial E_{x}}=\epsilon_{\infty} \frac{\partial P_{x}\left(D_{x}\right)}{\partial D_{x}}
$$

This relation is verified in Fig. S5.

Table S3: The optical dielectric constant of the liquid water calculated with PBE functional at constant $E_{x}$.

\begin{tabular}{c|ccc}
\hline & $E_{z}$ (TZV2P, GTH, 320 Ry) & $E_{z}$ (TZV2P, GTH, 520 Ry) & $E_{z}$ (PW, USPP, 200 Ry) [9] \\
\hline$\epsilon_{\infty}$ & 1.73 & 1.73 & 1.74 \\
\hline
\end{tabular}

Table S4: The optical dielectric constant of the liquid water calculated at constant $D_{x}$ and constant $E_{x}$ compared.

\begin{tabular}{c|cc}
\hline & $D_{z}$ (TZV2P, GTH, 320 Ry) & $E_{z}$ (TZV2P, GTH, 320 Ry) \\
\hline$\epsilon_{\infty}$ & 1.73 & 1.73 \\
\hline
\end{tabular}

\section{B.3 Structure of PBE Water at $\mathrm{E}=0$ and $\mathrm{D}=0$}

From our previous publication [4], we know that $\mathbf{E}=0$ and $\mathbf{D}=0$ are electrical boundary conditions which only affect the total polarization of the system. The structure of PBE water at $\mathbf{E}=0$ and $\mathbf{D}=0$ were found to be virtually identical. As in shown Fig. S6 this observation also applies to PBE water.

\section{References}

[1] E L Pollock, B J Alder, and G N Patey, "Static Dielectric-Properties of Polarizable Stockmayer Fluids", Physica A 108(1), pp. 14-26 (1981). 
[2] J.-M. Caillol, D Levesque, J J Weis, P G Kusalik, and G N Patey, "Computer simulation and theoretical results for a polar-polarizable fluid", Mol. Phys. 55, pp. 65-76 (1985).

[3] M Neumann and O Steinhauser, "Computer-Simulation and the Dielectric-Constant of Polarizable Polar Systems", Chem Phys Lett 106(6), pp. 563-569 (1984).

[4] C Zhang and M Sprik, "Computing the Dielectric Constant of Liquid Water at Constant Dielectric Displacement”, Phys. Rev. B 93, pp. 144201 (2016).

[5] Massimiliano Stengel, Nicola A Spaldin, and David Vanderbilt, "Electric displacement as the fundamental variable in electronic-structure calculations", Nat. Phys. 5(4), pp. 304308 (2009).

[6] A D Buckingham, "A Theory of Dielectric Polarization of Polar Substances", Proc. R. Soc. A 238, pp. 235-244 (1956).

[7] M S Wertheim, “Theory of Polar Fluids”, Mol. Phys. 36(4), pp. 1217-1240 (1978).

[8] P Ghosez, J P Michenaud, and X Gonze, "Dynamical atomic charges: The case of AB O 3 compounds", Phys. Rev. B 58(10), pp. 6224-6240 (1998).

[9] A Saitta, Franz Saija, and Paolo Giaquinta, "Ab Initio Molecular Dynamics Study of Dissociation of Water under an Electric Field", Phys. Rev. Lett. 108(20), pp. 207801 (2012).

[10] AK Soper, "The Radial Distribution Functions of Water and Ice from 220 to $673 \mathrm{~K}$ and at Pressures up to $400 \mathrm{MPa}$ ", Chem. Phys. 258(2), pp. 121-137 (2000). 

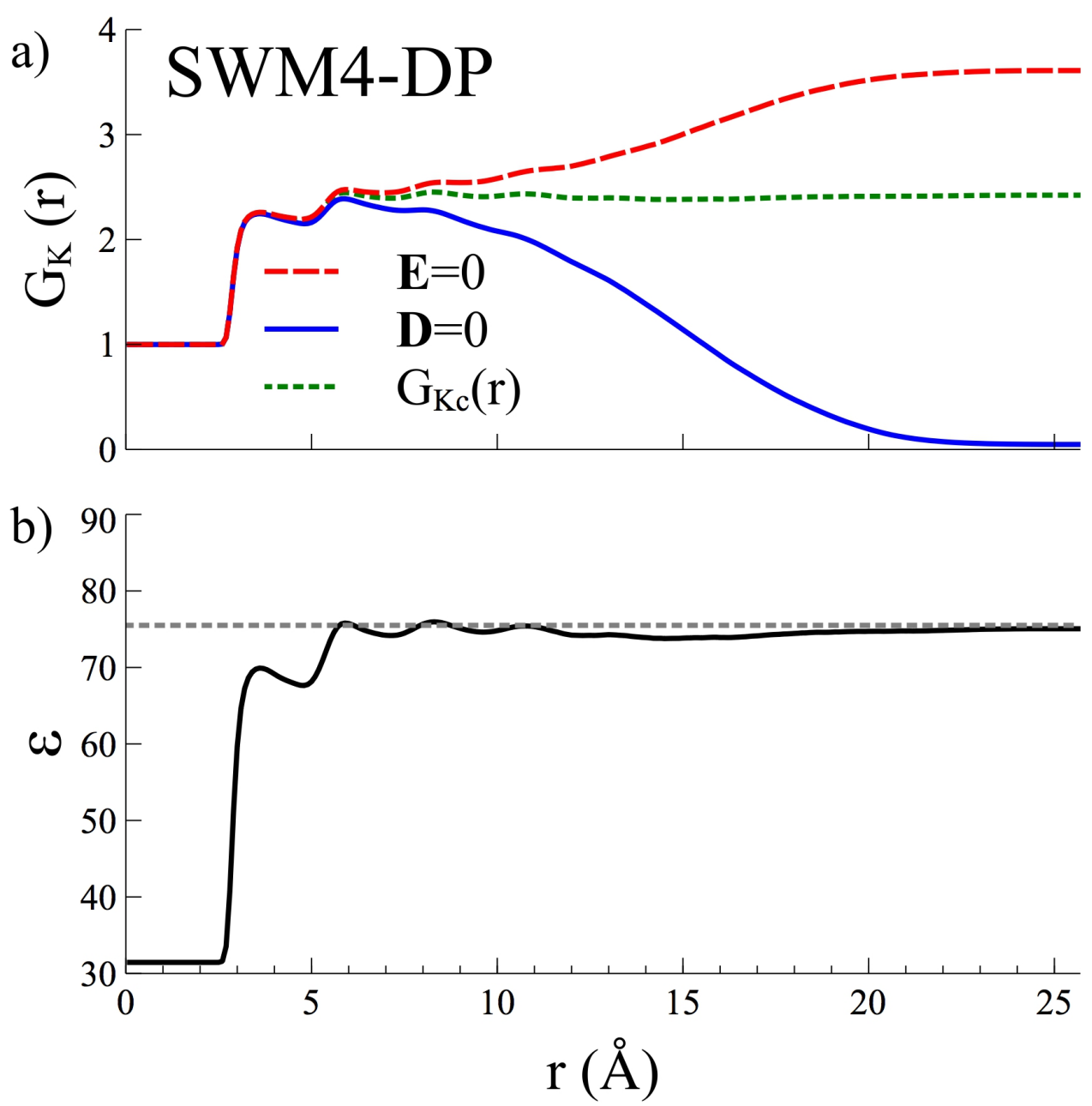

Figure S1: a) Kirkwood g-factor $G_{K}(r)$ of SWM4-DP water calculated at $\mathbf{E}=0$ (red curve) and $\mathbf{D}=0$ (blue curve) and the composite Kirkwood g-factor $G_{\mathrm{Kc}}(r)$ (green curve). b) The r-dependent dielectric constant $\epsilon(r)$ from the Kirkwood-Onsager formula using $G_{\mathrm{Kc}}(r)$ (solid curve) and the converged static dielectric constant $\epsilon$ calculated from volume dipole fluctuation at $\mathbf{E}=0$ (dashed line). $\epsilon_{\infty}$ as calculated for SWM4-DP is 1.48. 

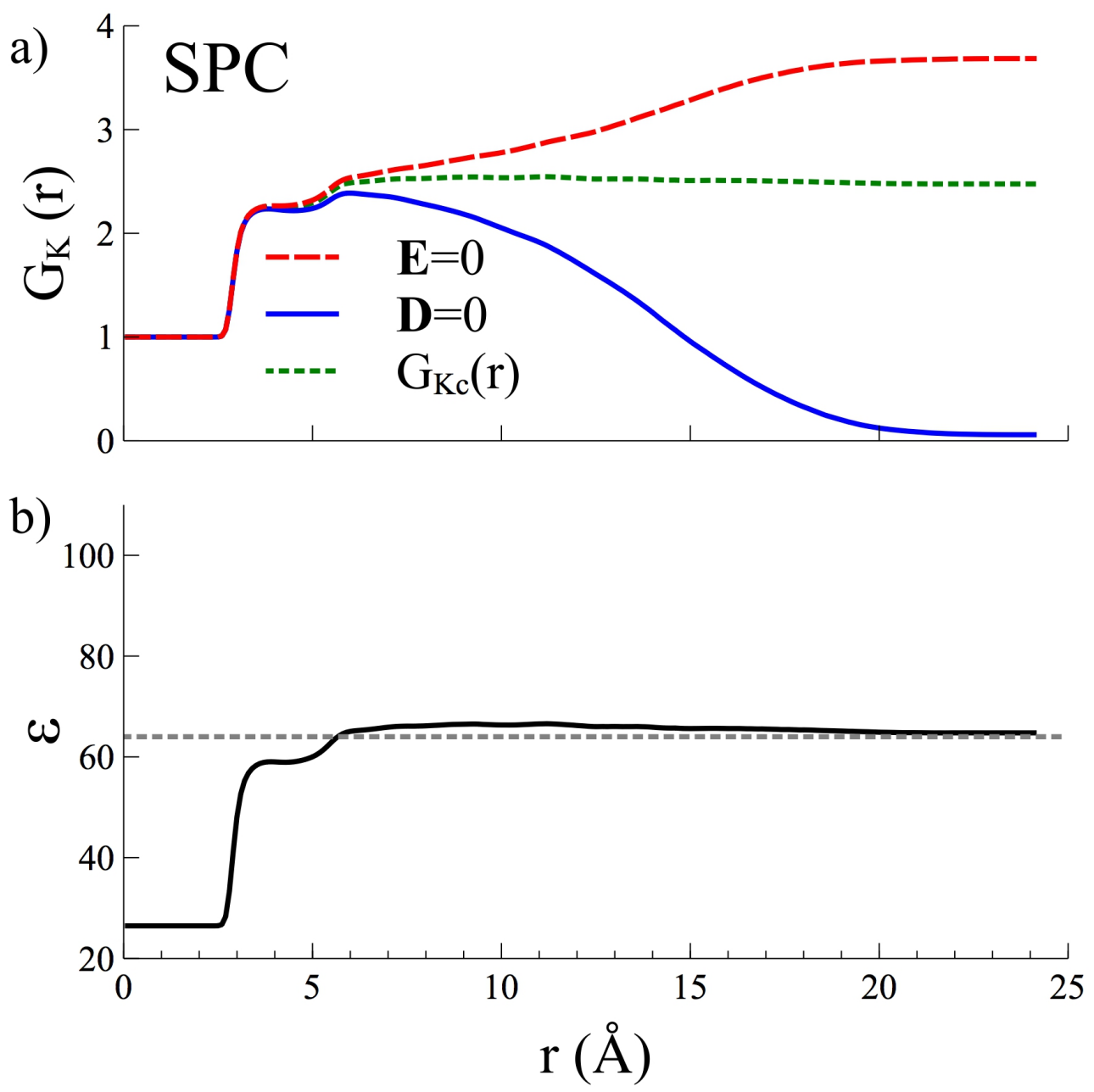

Figure S2: a) Kirkwood g-factor $G_{K}(r)$ of SPC water calculated at $\mathbf{E}=0$ (red curve) and $\mathbf{D}=0$ (blue curve) and the composite Kirkwood g-factor $G_{\mathrm{Kc}}(r)$ (green curve). b) The rdependent dielectric constant $\epsilon(r)$ from the Kirkwood-Onsager formula using $G_{\mathrm{Kc}}(r)$ (solid curve) and the converged static dielectric constant $\epsilon$ calculated from volume dipole fluctuation at $\mathbf{E}=0$ (dashed line). 

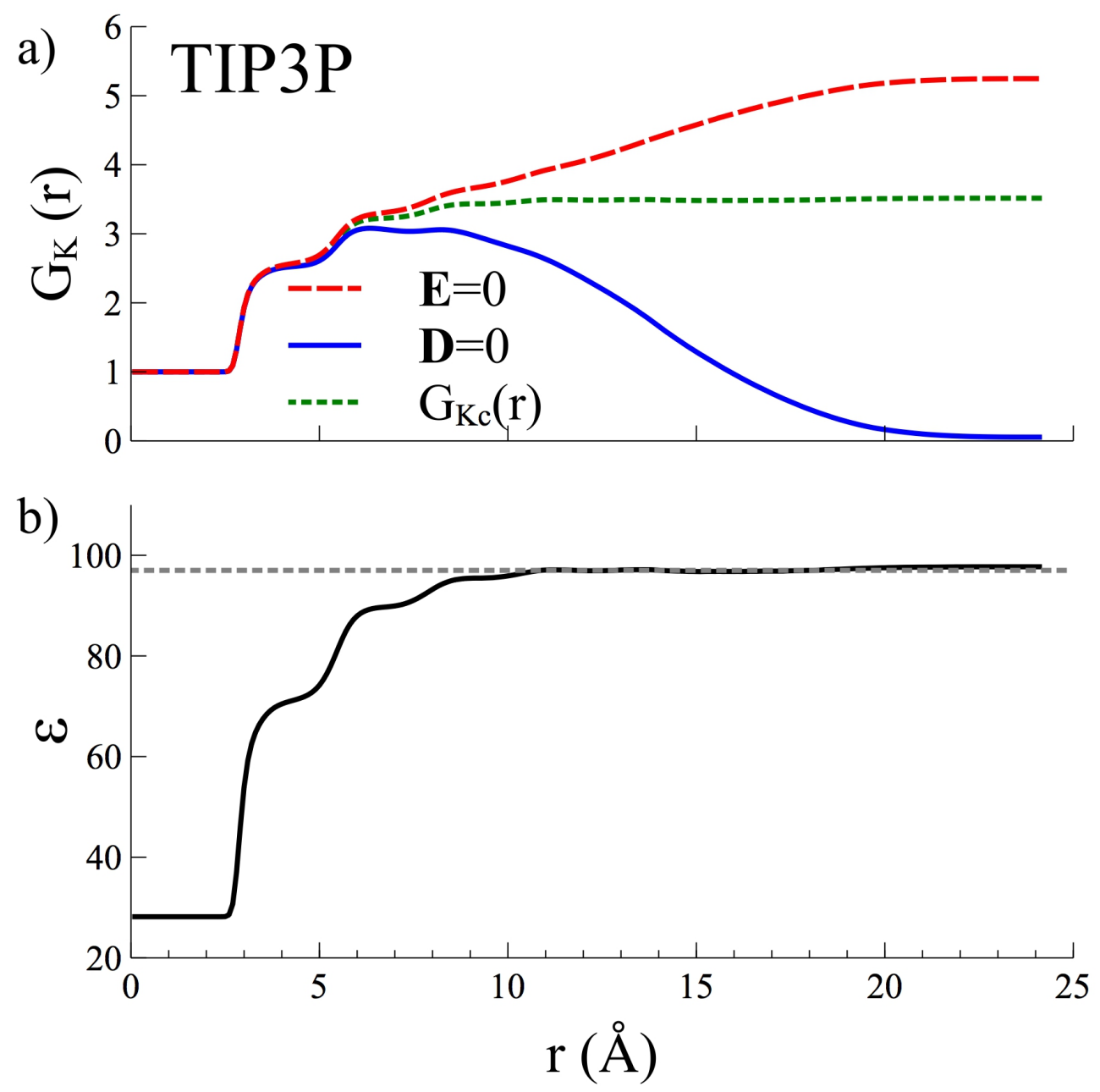

Figure S3: a) Kirkwood g-factor $G_{K}(r)$ of TIP3P water calculated at $\mathbf{E}=0$ (red curve) and $\mathbf{D}=0$ (blue curve) and the composite Kirkwood g-factor $G_{\mathrm{Kc}}(r)$ (green curve). b) The r-dependent dielectric constant $\epsilon(r)$ from the Kirkwood-Onsager formula using $G_{\mathrm{Kc}}(r)$ (solid curve) and the converged static dielectric constant $\epsilon$ calculated from volume dipole fluctuation at $\mathbf{E}=0$ (dashed line). 

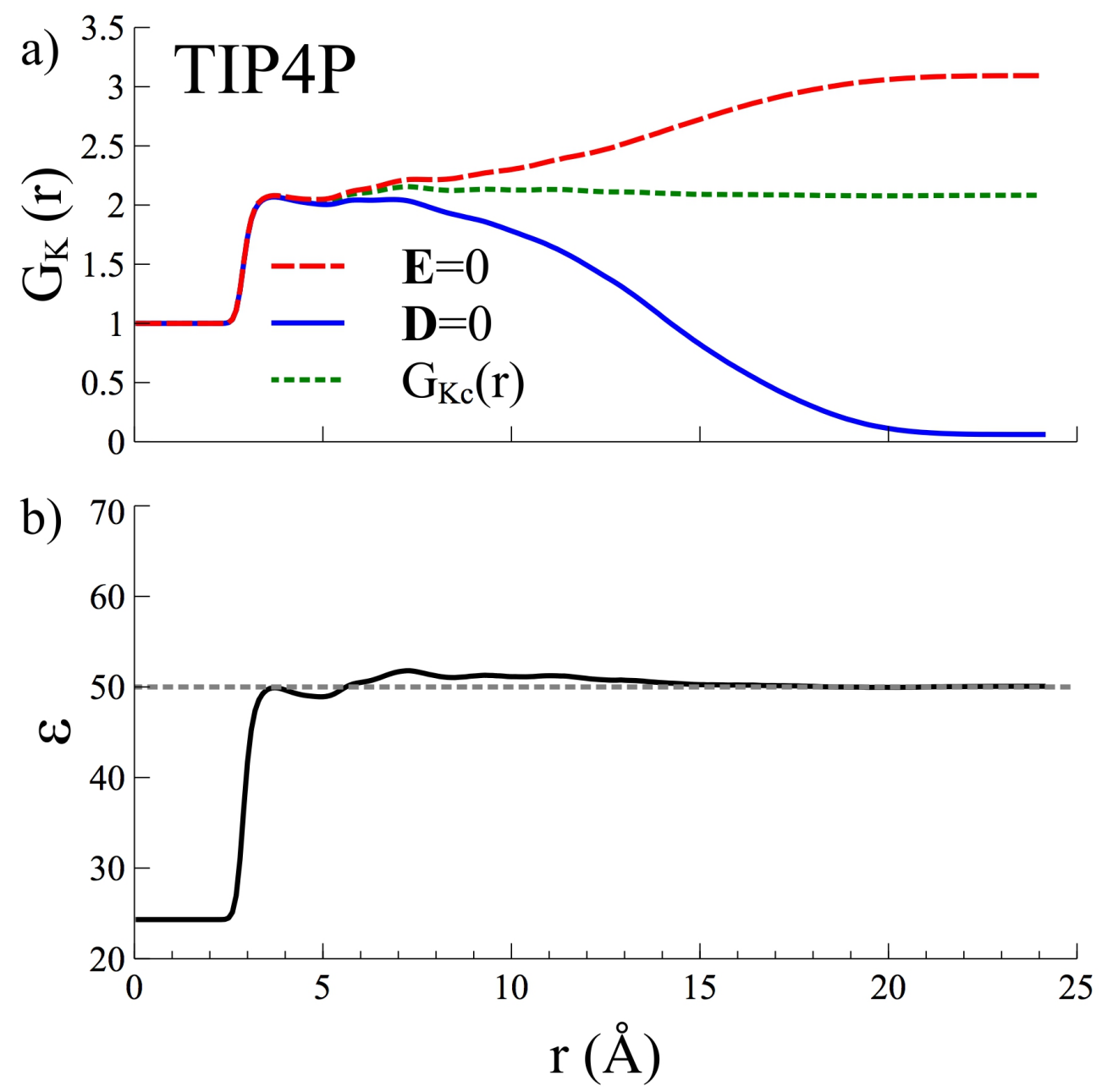

Figure S4: a) Kirkwood g-factor $G_{K}(r)$ of TIP4P water calculated at $\mathbf{E}=0$ (red curve) and $\mathbf{D}=0$ (blue curve) and the composite Kirkwood g-factor $G_{\mathrm{Kc}}(r)$ (green curve). b) The r-dependent dielectric constant $\epsilon(r)$ from the Kirkwood-Onsager formula using $G_{\mathrm{Kc}}(r)$ (solid curve) and the converged static dielectric constant $\epsilon$ calculated from volume dipole fluctuation at $\mathbf{E}=0$ (dashed line). 


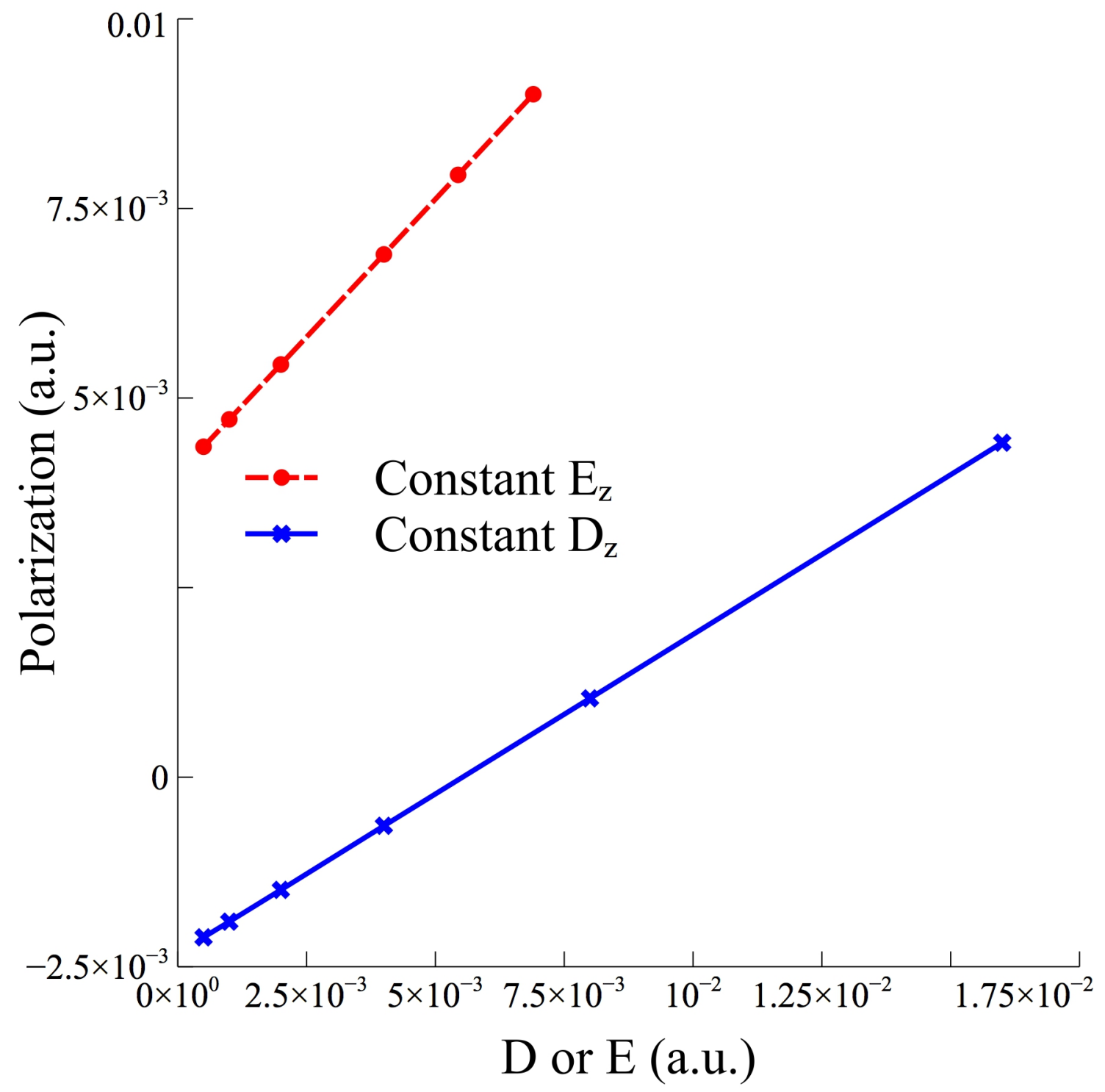

Figure S5: Polarization at fixed geometry as function of applied $E_{x}$ (red curve) or $D_{x}$ field (blue curve) in PBE bulk water. 


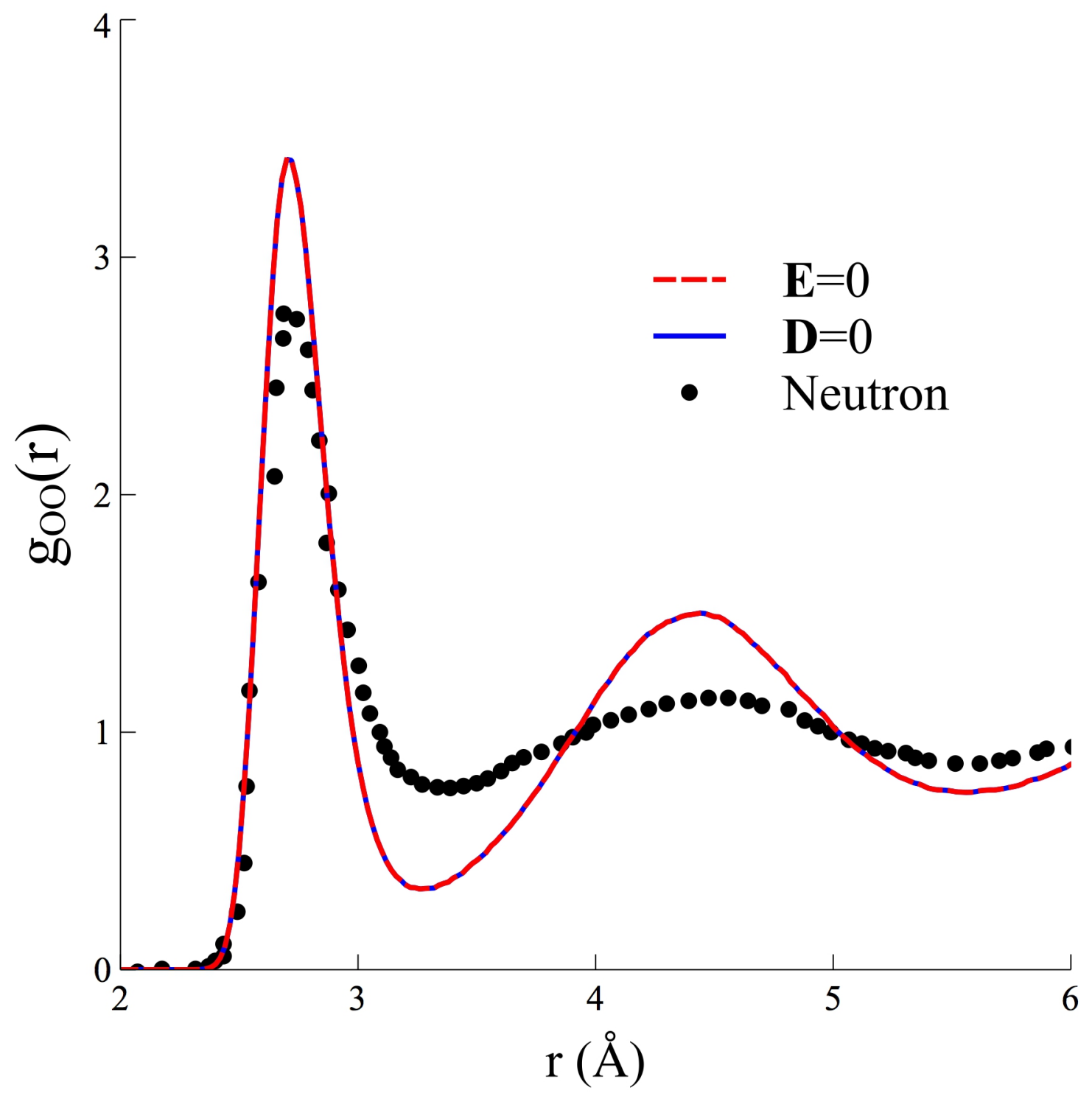

Figure S6: Oxygen-oxygen radial distribution function of PBE water at $\mathbf{E}=0$ (red curve) and $\mathbf{D}=0$ (blue curve), in comparison with experimental data at ambient condition [10]. 